\title{
Téoros
}

Revue de recherche en tourisme

\section{L'effet sur la vie familiale de l'action communautaire au sein du Mouvement québécois des camps familiaux}

\section{Rapport d'évaluation}

\author{
Louise Gaudreau, Louis Jolin et Geneviève Buissonnet-Verger
}

Volume 20, numéro 3, automne 2001

Comportements touristiques

URI : https://id.erudit.org/iderudit/1071628ar

DOI : https://doi.org/10.7202/1071628ar

Aller au sommaire du numéro

Éditeur(s)

Université du Québec à Montréal

ISSN

0712-8657 (imprimé)

1923-2705 (numérique)

Découvrir la revue

Citer cet article

Gaudreau, L., Jolin, L. \& Buissonnet-Verger, G. (2001). L'effet sur la vie familiale de l'action communautaire au sein du Mouvement québécois des camps familiaux : rapport d'évaluation. Téoros, 20(3), 21-23.

https://doi.org/10.7202/1071628ar 


\section{L'effet sur la vie familiale de l'action communautaire au sein du Mouvement québécois des camps familiaux \\ Rapport d'évaluation}

\section{Louise Gaudreau. Louis Jolin et Geneviève Buissonnet-Verger}

e présent article fait état d'une recher-

- che évaluative qui a permis d'étudier les consequences, sur la vie familiale de familles à revenus modestes, de l'action communataire qui se réalise au sein du Mouvenent québécois des camps familiaux (MQCF). Unique en son genre au Quebec, le MQCF existe depuis une vingtaine d'années. Il est constitué d'un réseau d'une quarantaine d'organismes communautaires répartis sur le territoire québécois; ce sont des associations familiales de départ en vacances et des corporations. gestionnaires de camps familiaux de vacances. En 1999, ces organismes pouvaient compter sur vingt-deux sites de séjour de vacances.

La recherche évaluative visait à estimer l'importance, pour la qualité de la vie familiale, du séjour de vacances sur ces sites pour familles à revenus modestes et à discerner les retombées significatives, sur ces familles, de leur intégration dans les groupes de départ en vacances ou dans les corporations gestionnaires de camps. Au regard de ces objectifs, la question à résoudre était la suivante: sous quels angles et jusqu'à quel point la vie familiale de familles a revenus modestes estelle favorisée : a) par le séjour en vacances familiales sur les sites et b) par la participation aux groupes de départ ou aux corporations gestionnaires? La demarche d'évaluation et la production des résultats ont été confiées à une équipe externe de chercheurs-évaluateurs de 1'UQAM. Le
MQCF et des responsables locaux des camps y ont aussi collaboré .

\section{La méthodologie}

On a retenu les indicateurs d'impact suivants : a) ce que les membres des familles constatent comme améliorations de leur vie familiale ; b) les relations de cause à effet, établies par les membres de la famille, entre le séjour en vacances ou l'engagement dans un groupe d'appartenance et les améliorations constatées ; c) la duréc anticipé ou rélle de ces améliorations, telle qu'estimée par les membres des familles.

L'évaluation était centrée sur l' utilisateur (client-centered). Les utilisateurs étaient des adultes et desenfants vacanciers. Leur point de vue a été prédominant dans les résultats de l'evaluation, ce qui, jumelé au partenariat entre l'équipe de l' UQAM et le MQCF dans la conception et la réalisation de cette évaluation, reflète l'orientation démocratique de l'évaluation.

Puisque les adultes et les enfants participants représentaient des familles et parlaient au nom de leur famille respective, chaque famille constituait un cas, ce quil permet d'affirmer que cette recherche a procédé par étude de cas multiples.

Les données ont été recueillies en utilisant une approche qualitative. Celle-ci s'est concrétisé par l'utilisation des techniques de focus growp, principalement, et de quelques observations participantes sur trois sites. Nous avions aussi prevu des entretiens de recherche individuels, mais ils n'ont pu être retenus comme moyen de collecte des donnés ${ }^{2}$.
Le focus group est une technique par « questions-réponses * en groupes de six à douze personnes animés par un intervieweur. Les instruments nécessaires à la collecte des données dans les focus groups ont été conçus et produits aux seules fins de cette évaluation. II s'agit pour l'essentiel d'un questionnaire, composé de trois grandes parties : une première présentait des questions générales sur les séjours en vacances, une autre portait sur l'organisation des vacances et une troisieme sur le séjour même.

Selon les disponibilites et les horaires convenus avec les responsables des camps et les participants volontaires, six focus groups d'adultes et cinq groupes d'enfants ont été evalués, pour un total de onze. Dans le cas des entants, aucun focus group n"a pu être réalise sur le sixiène site, car le nombre d'enfants consentants était insuffisant, Les focus groups d'adultes, un par site, prenaient environ une heure et trente minutes, allors que ceux des enfants duraient une quarantaine de minutes en moyenne. Le nombre de participants par focus group d'adultes variait de quatre à onze personnes selon le site et le nombre de participants par focus group d'enfants se situait de cinq à onze selon le site.

Compte tenu de l'annulation des entretiens individuels, l'observation participante a été introduite dans la démarche d'évaluation comme technique de prise de données sur trois sites. Elle a porté sur l'organisation générale du camp, les caractéristiques des activites en fonction de certains aspects liếs à l'évaluation, les membres des fámilles et leurs interactions, les relations entre les familles et entre les gens. Ces 
données ont servi à compléter, contextualiser et préciser les résultats des focus groups afin de faciliter leur interprétation. Par conséquent, l'observation participante s'est substituée à l'entretien de recherche individuel comme méthode de triangulation (corroboration des données obtenues au moyen des focus groups) pour accroître la certitude des résultats d'êvaluation.

Les données ont été soumises par la suite à une analyse de contenu par thèmes et, en partie, elles ont été analysées en suivant une approche de * théorisation ancrée $*$ (grounded theory) selon laquelle l'analyste cherche à dégager les grandes idées qui sont évoquées par les participants et qui caractérisent leurs propos. Puis, les résultats ont été analysés à nouveau et interprétés en fonction de critères d'évaluation.

Compte tenu des sites et des participants choisis, de leurs caractéristiques et de l'approche de l'évaluation, de sa méthodologie et des instruments de prise de données, on peut affirmer que le mode de production est valable. En effet, le nombre et la diversification des participants sont suffisants, ceux-ci sont représentatifs des vacanciers et des familles à revenus modestes qui fréquentent les camps familiaux et qui appartiennent aux regroupements communautaires, leur distribution entre les sites est suffisante pour illustrer les différents types de sites et de vacanciers, le nombre de focus groups est élevé et il y a triangulation par observation participante sur trois sites.

\section{Les résultats}

Les 48 adultes et les 39 enfants qui ont participé à la démarche d'évaluation séjournaient sur 6 des 22 sites de vacances du réseau, pendant une des périodes comprises entre le 20 juillet et le 22 août 1999. Trois des sites choisis sont situés à l'intérieur d'un rayon de 100 a 150 kilomètres de Montréal et les trois autres dans un rayon semblable autour de deux autres centres urbains (Québec et Sherbrooke), de façon à varier la provenance des participants à l'étude. Ces sites varient aussi par leur capacité d'accueil (nombre de familles possible par période de vacances), par l'ampleur ou la diversité des installations ainsi que par les types de groupes d'appartenance présents au camp.

Bien que les sites choisis soient à bonne distance de Montréal, la trés grande majorité $(85 \%)$ des participants à l'étude viennent de la région montréalaise, comme ce fut aussi observé dans des études antérieures ${ }^{3}$. Parmi les participants adultes, $38 \%$ en sont à plus de cinq séjours dans un camp familial, les autres se répartissant entre un premier, un deuxième, un troisième ou un quatrième séjour de vacances au camp. Le groupe d'âge le plus fréquent parmi les adultes est celui des 30-39 ans $(56 \%)$, suivi des $40-49$ ans $(28 \%)$. Près des trois quarts sont des femmes, ce qui correspond aux observations provenant d'études antérieures. La moyenne des revenus familiaux annuels est de 24330 \$; dans $42 \%$ des cas, ce revenu est inféricur à $15000 \$$. Un peu plus de la moitié des adultes vivent sans conjoint ou conjointe, quoique les trois quarts aient deux ou trois enfants. Pour la plupart, ces adultes étaient les parents des 39 enfants qui ont aussi participé à l'étude. Les garçons et les filles étaient en proportion à peu près équivalente, soit $: 52 \%$ de garçons et $48 \%$ de filles. Les jeunes de 14 ans constituaient $5 \%$ de l'ensemble, $36 \%$ avaient 12 ou 13 ans, $31 \%$ avaient 10 ou 11 ans et $28 \%$ étaient âgés de 9 ans.

Selon les résultats obtenus et reproduits au tableau 1 , la vie familiale est favorisée

\begin{tabular}{|c|c|c|c|c|}
\hline Interprataitio & $\begin{array}{r}\text { TA } \\
\text { 3 resulats e }\end{array}$ & $\begin{array}{l}\text { Al I } \\
\text { adion des c }\end{array}$ & feres devented & \\
\hline ANGLES & & & CRITERES & \\
\hline D AIVELIORARION & $\begin{array}{l}\text { (a) } \\
\text { proportion de } \\
\text { participants }\end{array}$ & $\begin{array}{c}\text { (b) } \\
\text { lien de } \\
\text { cause-effet }\end{array}$ & $\begin{array}{l}\qquad \mathrm{c}\rangle \\
\text { importance de } \\
\text { lamélioration }\end{array}$ & $\begin{array}{l}\text { (d) } \\
\text { persistance de } \\
\text { I'amélioration }\end{array}$ \\
\hline 1 - Par le séjour & & & & \\
\hline Relations parents-enfants & + & ++ & ++ & +- \\
\hline Vie de couple & ++ & + & + & $4=$ \\
\hline $\begin{array}{l}\text { Communications } \\
\text { et écoute de l'enfant }\end{array}$ & + & +4 & + & +- \\
\hline Discipline & + & ++ & + & +- \\
\hline Modeles parentaux & + & ++ & + & +- \\
\hline Individu: & & & & \\
\hline - État global & + & $+t$ & ++ & 0 \\
\hline - dév. soc. et appr. enfant & + & +4 & + & 4: \\
\hline - estime de soi & + & + & + & 4- \\
\hline - relations interpersonnelles & + & + & + & 0 \\
\hline - entraide & + & \#- & + & +: \\
\hline $\begin{array}{l}\text { - intégration soc. } \\
\text { des groupes particliers }\end{array}$ & + & H+ & +- & 4- \\
\hline $\begin{array}{l}\text { 2- Par participation au } \\
\text { groupe ou à la corporation }\end{array}$ & & & & \\
\hline Individu : & & & & \\
\hline - briser l'isolement & ++ & $4+$ & ++ & ++ \\
\hline * relations interpersonnelles & + & +4 & + & + \\
\hline * estime de soi & + & + & + & + \\
\hline - entraide & + & + & + & + \\
\hline $\begin{array}{l}\text { Influencas générales } \\
\text { sur la vie de famille }\end{array}$ & +- & +- & +- & +- \\
\hline
\end{tabular}

\section{LÉGENDE:}

++ signifie beaucoup; $\quad+$ - signifie a la limite; 0 signitie absence; - signifie a lencontre du critère
+ signifie suffisamment; 
sous plusieurs angles pendant le séjour de vacances au camp. Au premier chef, la relation parent-enfant, la vie de couple et un aspect individuel, soit l'état global des membres de la famille pris séparément, se trouvent améliorés, Puis, sur un deuxième plan, les ambliorations se font ressentir sur l'exercice de la discipline parentale envers l'enfant et sur un autre aspect individuel, soit le developpement social et les apprentissages effectués par les enfants pris séparément. Un autre aspect individuel, voire interindividuel, se trouve au troisieme rang des améliorations identifiées: il s"agit des relations interpersonnelles entre les adultes et entre les enfants de familles differrentes, Viennent ensuite, au quatrième rang, des améliorations de la communication et de l'ccoute de l'enfant. par les parents, de la connaissance de modèles parentaux diversifiés et, encore sur le plan individuel, la facilitation de l'intégration sociale de personnes appartenant à des groupes particuliers (par exemple: ethnies minoritaires ou individus intellectuellement handicapes). Enfin, le cinquième rang est occupé par un aspect très interindividuel, celui de l'entraide entre les adultes et entre les enfants de familles différentes.

Peu d'eléments ont été évoqués par les participants au regard de la deuxiême partie de la question soulevée, soit l'effet de l'appartenance à un regroupement (groupe de depart en vacances ou corporation gestionnaire). D'abord, sur le plan individuel ou interindividuel, cette appartenance brise l'isolement, promeut les relations interpersonnelles, l'estime de soi et l'entraide. Ensuite seulement, elle favorise un peu la vie familiale, mais globalement et sans que les participants n'aient de points précis à telever sur cet aspect.

En somme, plusicurs améliorations importantes de la vie familiale ont été constatées. mais elles n'ont que peu de suites après la fin de la periode de vacances au camp. La seule exception est la persistance d'un effet attribué à l'appartenance à un regroupement, celui de briser l'isolement entre les adultes de familles différentes.
Dans le but de poser un jugement sur l'ampleur de l'effet de l'action communautaire au sein du MQCF, nous avons appliqué aux résultats les critères d'évaluation suivants : la proportion des répondants des divers. types de groupes participants qui :

1)identifient les mêmes aspects de la vie familiale améliorés grâce au séjour de vacances au camp ou grâce à l appartenance au regroupement ;

2)lient l'amélioration de certains aspects de la vie familiale à leur séjour au camp ou a leur participation aux groupes de départ en vacances ou â une corporation gestionnaire de camps familiaux :

3)trouvent que des aspects de leur vie familiale ont été grandement améliorés grâce au séjour sur le site ou à leur participation au groupe de départ ou à la corporation ;

4)indiquent que les aspects améliorés le sont pour une période de temps qui dépasse la durée du séjour au camp.

En conséquence, le jugement rendu est le suivant. L'effet, sur la vic familiale des familles à revenus modestes, de $\mathrm{l}^{5}$ action communautaire au sein du MQCF est positif et significatif. Il reste cependant modéré en raison du peu de persistance des améliorations constatées et parce que la participation au groupe de départ ou à une corporation gestionnaire n ${ }^{\prime}$ est que peu liée à la vie familiale en tant que telle. Cependant, sans l'appartenance à ces regroupements, point de séjour au camp et aucune retombée positive possible de ce séjour sur la vie familiale. Les recommandations émises à la suite de cette recherche evaluative proposent au MQCF différentes avenues pour augmenter son effet dejà positif et significatif sur la vie familiale des familles ả revenus modestes. Pour être en mesure de donner suite à ces recommandations, il faudra toutefois que le MQCF accroisse ses moyens d'action ; en corollaire, cela exige qu'il accroisse ses moyens financiers.
Louise Gaudreau est professene au Departement des sciences de l'éducation et chercheure à l'Wnstitut Santé et Société de I'UQAM - Louis Jolin est profes. sear au Departement d'etudes urbames et touristiques de l'UOAM et nembre du Comité de rédaction de la revue Teoros: Geneviève Buíssonnet-Verger est assistante de recherche au Département des sciences de l'education et au CIRADE dE I'UQAM.

\section{Notes}

1 Outre les auteurs du present article, ont participe à l'evaluation Mmes Cécile Baillargeon, Judith Gaudet et M. JeanPierre Jodoin, étudiants au doctorat ; des representants du Mouvement québecois des camps familiaux, soit MM. Martin Plourde, Alain Dupont et Lue Boily ; les responsables locaux des camps ainsi que M. Michel Lizée, agent de développement au Service aux collectivités de l'UQAM. Les fonds qui ont permis de réaliser cette evaluation provenaient du Secrétariat à l'action communautaire autonome (SACA) du Gouvernement du Québec et du Service aux collectivités de l'UQAM.

2 Compte tenu du contexte de vacances et de la planification des activites sur le site des camps, il a êté impossible à l'equipe d'évaluation de réaliser les entretiens de recherche individuels prévus initialement. Les réponses provenant des quelques rares entretiens individuels qui ont néanmoins été réalisés (trois enfants, sur le sixième site, à partir du questionnaire des focus groups et deux adultes), ont été intégrees, aux fins d'analyse, aux résultats des focus groups.

3 Une recherche reposant sur les methodes quantitatives a été réalisécen 1998 par Jean Stafford et Bruno Sarrasin. Elle a pour titre : Ende des besoins et mesure de la satiffaction des usagers dans les etablissements membres du Mouvement quebecois des camps familaw. Eudes, materiaux er documens 12. Montreal: UQAM, Departement d'études urbaines et touristiques. Cette étude référait à une autre réalisé dix ans auparavant, soit en 1987. 